\title{
Psicologia em Revista
}

Pontifícia Universidade Católica de Minas Gerais 


\section{CONSELHO EDITORIAL NACIONAL}

\section{Brasil:}

Antônio Roazzi - Universidade Federal de Pernambuco - UFPE

Cláudio S. Hutz - Universidade Federal do Rio Grande do Sul - UFRGS

Francisco M. C. Martins - Universidade de Brasília - UnB

Joseli Bastos da Costa - Universidade Federal da Paraíba - UFPB

Lino de Macedo - Universidade de São Paulo - USP

Marco Aurélio Máximo Prado - Universidade Federal de Minas Gerais - UFMG

Maria Lucia Boarini - Universidade Estadual de Maringá - UEM

Norma M. Takeuti - Universidade Federal do Rio Grande do Norte - UFRN

Paulo César Carvalho Ribeiro - Universidade Federal de Minas Gerais - UFMG

Paulo Rogério M. Menandro - Universidade Federal do Espírito Santo - UFES

Sônia Margarida Gomes de Sousa - Universidade Católica de Goiás - UCG

Teresa Cristina O. Carreteiro - Universidade Federal Fluminense - UFF

\section{Exterior:}

Alfredo Zenoni - Section Clinique Bruxelles - Paris VIII - Bélgica

Mireille Cifalli - Université de Génève - Suíça

Patrícia Álvarez - Universidad de Buenos Aires - UBA - Argentina

Raúl Rocha Romero - Universidad Nacional Autónoma de México - Unam - México

Robert Sevigny - Université de Montreal - Canadá

Mireille Cifalli - Université de Génève - Suíça

Patrícia Álvarez - Universidad de Buenos Aires - UBA - Argentina

Raúl Rocha Romero - Universidad Nacional Autónoma de México - Unam - México

Robert Sevigny - Université de Montreal - Canadá 
DIRETORA DO INSTITUTO DE PSICOLOGIA - PUC MINAS:

Maria Cristina Martins de Andrade

\section{COMISSÃO EDITORIAL}

Prof. a Dr. ${ }^{a}$ Ilka Franco Ferrari, PUC Minas, Brasil

Prof. ${ }^{a}$ Dr. ${ }^{a}$ Jacqueline de Oliveira Moreira, PUC Minas, Brasil

Prof. ${ }^{a}$ Dr. ${ }^{a}$ Roberta Carvalho Romagnoli, PUC Minas, Brasil

\section{REVISÃO:}

Alessandro Faleiro Marques (português)

José Ruiz Guillén (espanhol)

Joseph John Dempsey (inglês)

\section{DIAGRAMAÇÃO:}

Evandro Ornelas

\section{ESTAGIÁRIA DE EDITORIA:}

Luiza Reis Machado, PUC Minas, Brasil

\section{INDEXADORES:}

Base de dados Qualis / Capes

Clase (Universidad Autónoma de México)

ClasePeriodica.htm

Icap (Indexação compartilhada de artigos de periódicos da rede Pergamum)

IndexPsi (CFP / PUC Campinas)

Latindex

Lilacs (Literatura latino-americana e do Caribe em ciências da saúde)

PePSIC / BVS-Psi (Portal de periódicos eletrônicos em Psicologia)

ULrich's

Base de Dados de Psicologia - Psicodoc

Directory of Open Access Journals - DOAJ 


\section{ADMINISTRAÇÃO SUPERIOR DA PONTIFÍCIA UNIVERSIDADE CATÓLICA DE MINAS GERAIS}

Grão-chanceler: Dom Walmor Oliveira de Azevedo

Reitor: Professor Dom Joaquim Giovani Mol Guimarães

Vice-reitora: Professora Patrícia Bernardes

Assessor especial da reitoria: Professor José Tarcísio Amorim

Chefe de gabinete do reitor: Professor Paulo Roberto de Sousa

Secretário-geral: Professor Ronaldo Rajāo Santiago

Pró-reitores e secretários: Planejamento e Desenvolvimento Institucional - Professor Carlos Barreto Ribas; Graduação - Professora Maria Inês Martins; Pesquisa e Pós-graduação - Professor Sérgio de Morais Hanriot; Extensão - Professor Wanderley Chieppe Felippe; Gestão Financeira - Professor Paulo Sérgio Gontijo do Carmo; Logística e Infraestrutura - Professor Rômulo Albertini Rigueira; Recursos Humanos - Professor Sérgio Silveira Martins; Diretor Executivo de Recursos Humanos - Prof. Eudes Weber Porto; Cultura e Assuntos Comunitários - Professora Maria Beatriz Rocha Cardoso; Comunicação - Professor Mozahir Salomão Bruck

Pró-reitores adjuntos dos campi e unidades: São Gabriel - Professor Miguel Alonso de Gouvêa Valle; Barreiro - Professor Renato Moreira Hadad; Contagem - Professor Maria José Viana Marinho de Mattos; Betim - Professor Eugenio Batista Leite; Arcos - Professor Marcelo Leite Metzker; Serro e Guanhães Professor Ronaldo Rajāo Santiago; Poços de Caldas - Professor Iran Calixto Abrão

\section{Missão}

Psicologia em Revista, periódico da Faculdade de Psicologia da Pontifícia Universidade Católica de Minas Gerais (PUC Minas), abre-se às diversas tendências teóricas e às mais variadas práticas, clássicas ou emergentes, vigentes nos campos da Psicologia. Publicação quadrimestral, Psicologia em Revista é bilíngue e publica em português e espanhol. Os textos em outras línguas, quando aceitos, serão traduzidos para o português.

ISSN: 1678-9563

Submissão de textos e orientações para autores:

http://periodicos.pucminas.br/index.php/psicologiaemrevista/about/submissions\#authorGuidelines

Outras informaçôes: http://periodicos.pucminas.br/index.php/psicologiaemrevista/about/

\section{Endereço eletrônico}

Psicologia em Revista: http://periodicos.pucminas.br/index.php/psicologiaemrevista/index

Correio eletrônico: psirevista@pucminas.br

\section{Endereço para correspondência}

Avenida Itaú, 525 - Prédio Redentoristas - $1^{0}$ subsolo - Bairro Dom Bosco, Belo Horizonte-MG, Brasil. CEP: 30730-280.

Telefone: (31) 3319-4922 


\section{Sumário}

Editorial

\section{ARTIGOS / ARTICLES / ARTÍCULOS}

Fenótipo neuropsicológico de crianças com síndrome de Down

Rosália Carmen de Lima Freire

Nietsnie de Souza Duarte

Izabel Hazin

O desenvolvimento da habilidade de assertividade e a convivência na escola:

relato de experiência

Denise da Silva Maia,

Marcela Bortolini

A inserção do psicólogo na residência multiprofissional em saúde:

um relato de experiência em oncologia

Jamile Luz Morais

Emily Suelen Antunes de Castro

Airle Miranda de Souza.....

O espaço de habitação e sua importância para a produção de subjetividade

Renato Ferreira Franco

Cornelis Johannes van Stralen

Mulher e trabalho: visibilizando o tecido e a trama que engendram o assédio moral

Bruna Meurer Antunes

Mary Sandra Carlotto

Marlene Neves Strey.

Errância e isolamento: as dimensões de desejo e de gozo da solidão

Miriam Debieux Rosa

Isabel Tatit.

O fantasiar metapsicológico do psicanalista e aquele do escritor criativo: semelhanças e distinções

Ricardo Lincoln Laranjeira Barrocas,

Osvaldo Costa Martins.

\begin{tabular}{|l|l|ll|}
\hline Psicologia em Revista & Belo Horizonte & p. 352-539 dez. 2012 \\
\hline
\end{tabular}


Corpos em revista: a construção de padrões de beleza na Vogue Brasil

Fernanda Lyrio Heinzelman

Bruna Krimberg von Mublen

Cristiane dos Santos Schleiniger

Madalena Dornelles Pereira Leite

Marlene Neves Strey...

Os processos de subjetivação de deficientes intelectuais no mercado

de trabalho: uma cartografia

Carlos Alberto Ribeiro

Roberta Carvalho Romagnoli...

Processos de identidade social e exclusão racial na infância

Thais Augusta Cunha de Oliveira Máximo

Leoncio Francisco Camino Rodriguez Larrain

Aline Vieira de Lima Nunes

Samuel Lincoln Bezerra Lins.

\section{SEÇÃO ABERTA / OPEN SECTION / SECCIÓN ABIERTA}

Resenha: A prática da terapia cognitivo-comportamental baseada em mindfulness e aceitação

Micheli Aparecida Gomes dos Santos.

Resumo de tese: Saúde mental na atenção básica: política, trabalho e subjetividade

Patricia Pinto de Paula

Resumo de dissertação: Um estudo teórico-clínico sobre o ato cerimonial em um caso de neurose obsessiva

Henrique Guilherme Scatolin....

Nominata dos consultores ad hoc (2012)

\begin{tabular}{|l|l|ll}
\hline Psicologia em Revista & Belo Horizonte & p. 352-539 dez. 2012
\end{tabular}

